\title{
COPD: Höheres Risiko für plötzlichen Herztod
}

\author{
US-Kardiologen identifizieren in einer epidemiologischen Kohorte die COPD als einen unabhän- \\ gigen Risikofaktor für den plötzlichen Herztod. Betablocker könnten das Risiko mindern.
}

Die wichtigste Todesursache bei COPD-Patienten sind kardiovaskuläre Ereignisse. Nicht völlig geklärt ist bisher, ob das ausschließlich auf eine erhöhte Inzidenz der koronaren Herzerkrankung (KHK) unter COPD-Patienten zurückgeht oder ob möglicherweise KHK-unabhängig auch das Risiko von lebensbedrohlichen Herzrhythmusstörungen erhöht ist.

Dass Letzteres der Fall sein könnte, dafür spricht eine epidemiologische Studie aus dem Kontext der „Oregon Sudden Unexpected Death Study“ [1] Für die Analyse wurden 728 Teilnehmer, die im Studienzeitraum einen plötzlichen Herztod erlitten hatten, 548 Kontrollprobanden mit KHK zugeordnet, die jeweils in derselben geografischen Region leben mussten. Ein weiteres Matching zwischen Indexpatienten und Kontrollprobanden erfolgte nicht. Diese Zuordnung erlaubt es, Risikofaktoren zu identifizieren, die über die bei plötzlichem Herztod in aller Regel vorliegende KHK hinausgehen. In der aktuellen Publikation war dies die COPD: 30,8 \% der Patienten mit plötzlichem Herztod, aber nur 12,8\% der KHK-Patienten ohne plötzlichen Herztod hatten eine COPD $(\mathrm{p}<0,0001)$.

Für Asthma bronchiale fand sich kein derartiger Unterschied. Patienten mit plötzlichem Herztod nahmen signifikant häufiger kurzwirksame, inhalative Betamimetika (22,3 vs. $12,6 \%$; $\mathrm{p}<0,0001)$ und geringfügig seltener Betablocker ein (60,6 vs. $66,4 \% ; p=0,03)$. In der u. a. für Medikation, Alter, Diabetes und Zigarettenkonsum adjustierten, multivariaten Analyse blieb das Risiko für plötzlichen Herztod bei Vorliegen einer (KHK mit) COPD mehr als doppelt so hoch wie bei Probanden mit KHK, aber ohne COPD. Kurzwirksame Betaagonisten erhöhten demnach nur bei jenen COPD-Patienten, die keine Betablocker einnahmen, das Risiko für einen plötzlichen Herztod.

\section{Rechtsventrikuläres Remodelling als Mechanismus?}

Die Autoren folgern aus ihren Ergebnissen, dass es einen von der KHK unabhängigen Zusammenhang zwischen COPD und plötzlichem Herztod geben müsse, der evtl. durch Betablockertherapie abgemildert werden kann. Als Mechanismus komme

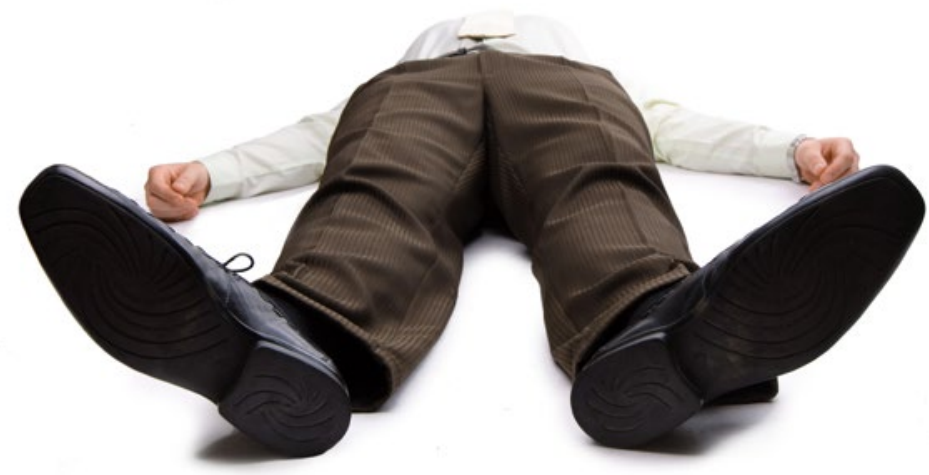

u. a. ein durch die COPD induziertes, rechtsventrikuläres Remodelling in Frage. Auch die COPD begleitende Entzündungsprozesse könnten eine Rolle spielen.

Vor dem Hintergrund dieser Daten wird in einem begleitenden Editorial diskutiert, ob es Sinn machen könnte, die COPD bei der Risikostratifizierung für den plötzlichen Herztod zu berücksichtigen, um die Indikationsstellung für einen ICD zu optimieren.

Die Kommentatoren weisen auch darauf hin, dass die Daten Fragen hinsichtlich der kardialen Sicherheit kurzwirksamer Betamimetika aufwerfen. Laufende randomisierte Studien werden hier neuen Diskussionsstoff liefern. Möglicherweise ist der Betablocker gerade KHK-Patienten mit COPD besonders wichtig.

Phillip Grätzel von Grätz

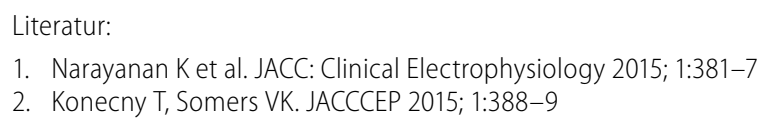

\section{Warum sind COPD-Kranke infektanfälliger?}

Wissenschaftler des Helmholtz Zentrums München haben in einem internationalen Team in Zusammenarbeit mit Kollegen vom Klinikum der Universität München und dem Deutschen Zentrum für Lungenforschung (DZL) gezeigt, dass Zigarettenrauch die Aktivität des Immunoproteasoms vermindert (Am J Respir Crit Care Med. 2016; online 12. Januar).

Zudem weisen COPD-Patienten verringerte Immunoproteasom-Level auf. Dies könnte dazu beitragen, dass COPD-Patienten anfälliger für Atemwegsinfekte sind, teilt das Helmholtz Zentrum München mit. Das Immunoproteasom ist eine Struktur in Säugetierzellen und dient dem Abbau von zellfremden Eiweißmolekülen, wie sie etwa bei einer Virusinfektion vorliegen. Seine Funktion ist mit einem zellulären Schredder vergleichbar. Die dabei entstehenden Proteinstücke werden gezielt dem Immunsystem präsentiert, welches spezifische Abwehrmaßnahmen vorbereitet.

Das Forscherteam um PD Dr. Silke Meiners vom Institut für Lungenbiologie (iLBD) / Comprehensive Pneumology Center (CPC) am Helmholtz Zentrum München fand heraus, dass Rauchen diesen Schutzmechanismus des Immunsystems entscheidend behindert. „Bei Experimenten mit Immunzellen konnten wir beobachten, dass Zigarettenrauch die Aktivität des Immunoproteasoms vermindert", erklärt die Erstautorin Ilona Kammerl. „Dadurch funktioniert das Präsentieren der zerkleinerten Proteinschnipsel gegenüber dem Immunsystem schlechter und die spezifische Immunantwort wird abgeschwächt." Zudem wiesen Lungen von COPDPatienten geringere Mengen an Immunoproteasomen auf. „COPD-Patienten erleben bei einer viralen Infektion häufig eine akute Verschlechterung der Lungenfunktion, von der sie sich oft nicht vollständig erholen. Dies deutet darauf hin, dass die spezifische Immunantwort gegenüber viralen Erregern in diesen Patienten vermindert ist. Unsere Daten weisen erstmals darauf hin, dass dies mit einer durch Zigarettenrauch verminderten Immunoproteasom-Aktivität zusammenhängt", unterstreicht Meiners.

Quelle: Pressemitteilung des Helmholtz Zentrum München 